\title{
Sirolimus induces apoptosis and reverses multidrug resistance in human osteosarcoma cells in vitro via increasing microRNA-34b expression
}

Yan ZHOU ${ }^{1, \#}$, Rui-hua ZHAO ${ }^{2, \#, ~ K u o-F u ~ T S E N G ~}{ }^{4}$, Kun-peng LI $^{1}$, Zhi-gang LU ${ }^{3}$, Yuan LIU ${ }^{3}$, Kun HAN ${ }^{1}$, Zhi-hua GAN ${ }^{1}$, Shu-chen $\mathrm{LIN}^{1}$, Hai-yan HU ${ }^{1, *}$, Da-liu MIN, *

${ }^{1}$ Department of Oncology, Shanghai Jiao Tong University Affiliated Sixth People's Hospital, Shanghai 200233, China; ${ }^{2}$ Department of Oncology, the First Affiliated Hospital of Zhengzhou University, Zhengzhou 450052, China; ${ }^{3}$ Department of Hematology, Zhujiang Hospital Affiliated with Southern Medical University, Guangzhou 510282, China; ${ }^{4}$ Department of Biophysics, Oregon State University, ALS2139 Corvallis, OR 97330, USA

Aim: Multi-drug resistance poses a critical bottleneck in chemotherapy. Given the up-regulation of mTOR pathway in many chemoresistant cancers, we examined whether sirolimus (rapamycin), a first generation mTOR inhibitor, might induce human osteosarcoma (OS) cell apoptosis and increase the sensitivity of OS cells to anticancer drugs in vitro.

Methods: Human OS cell line MG63/ADM was treated with sirolimus alone or in combination with doxorubicin (ADM), gemcitabine (GEM) or methotrexate (MTX). Cell proliferation and apoptosis were detected using CCK-8 assay and flow cytometry, respectively. MiRNAs in the cells were analyzed with miRNA microarray. The targets of miR-34b were determined based on TargetScan analysis and luciferase reporter assays. The expression of relevant mRNA and proteins was measured using qRT-PCR and Western blotting. MiR34, PAK1 and ABCB1 levels in 40 tissue samples of OS patients were analyzed using qRT-PCR and in situ hybridization assays. Results: Sirolimus $(1-100 \mathrm{nmol} / \mathrm{L})$ dose-dependently suppressed the cell proliferation $\left(\mathrm{IC}_{50}=23.97 \mathrm{nmol} / \mathrm{L}\right)$ and induced apoptosis. Sirolimus $(10 \mathrm{nmol} / \mathrm{L})$ significantly sensitized the cells to anticancer drugs, leading to decreased IC ${ }_{50}$ values of ADM, GEM and MTX (from 25.48, 621.41 and $21.72 \mu \mathrm{mol} / \mathrm{L}$ to $4.93,73.92$ and $6.77 \mu \mathrm{mol} / \mathrm{L}$, respectively). Treatment of with sirolimus increased miR-34b levels by a factor of 7.5 in the cells. Upregulation of miR-34b also induced apoptosis and increased the sensitivity of the cells to the anticancer drugs, whereas transfection with miR-34b-AMO, an inhibitor of miR-34b, reversed the anti-proliferation effect of sirolimus. Two key regulators of cell cycle, apoptosis and multiple drug resistance, PAK1 and ABCB1, were demonstrated to be the direct targets of miR-34b. In 40 tissue samples of OS patients, significantly higher miR-34 ISH score and lower PAK5 and ABCB1 scores were detected in the chemo-sensitive group.

Conclusion: Sirolimus increases the sensitivity of human OS cells to anticancer drugs in vitro by up-regulating miR-34b interacting with PAK1 and ABCB1. A low miR-34 level is an indicator of poor prognosis in OS patients.

Keywords: osteosarcoma; sirolimus; doxorubicin; gemcitabine; methotrexate; chemosensitivity; miR-34b; PAK1; ABCB1

Acta Pharmacologica Sinica (2016) 37: 519-529; doi: 10.1038/aps.2015.153; published online 29 Feb 2016

\section{Introduction}

Osteosarcoma (OS) is the most common primary malignant bone tumor. The 5-year survival rate of osteosarcoma was less than $20 \%$ during the initial half of the 20th century. However, with the newly introduced multi-agent chemotherapy and

\footnotetext{
\# These authors contributed equally to this work.

* To whom correspondence should be addressed.

E-mail xuri1104@163.com (Hai-yan HU); mindaliu69@126.com (Da-liu MIN)

Received 2015-08-25 Accepted 2015-12-29
}

advances in surgical techniques in the 1970s, the long-term survival of patients with localized osteosarcoma has improved to approximately $60 \%$. Chemotherapy is fundamental to cancer management and is responsible for most cases of adjuvant treatment in patients following surgical procedures ${ }^{[1]}$. However, no major changes in chemotherapy for metastatic diseases have occurred in the past 30 years. Multiple drug resistance (MDR) is the greatest obstacle to the overall survival of patients with metastatic disease. The processes leading to MDR are complex. Most data regarding the mechanisms of resistance have been derived from in vitro models of cells 
selected by exposure to high drug concentrations. Given the up-regulation of the mTOR pathway in many chemoresistant cancer ${ }^{[2]}$, mTOR inhibitors provide a logical solution to re-sensitize tumor cells to chemotherapy or to delay the development of resistance to treatment. Recent studies have demonstrated the effectiveness of sirolimus (rapamycin) in solid tumors, such as metastatic breast ${ }^{[3]}$, pancreatic ${ }^{[4]}$, and renal carcinomas ${ }^{[5]}$. Sirolimus is a first generation mTOR inhibitor ${ }^{[6-8]}$. Sirolimus also can function as an MDR reversal agent ${ }^{[9,10]}$. Based on several findings ${ }^{[11]}$, we hypothesized that sirolimus would induce OS cell apoptosis and increase the sensitivity of the OS cell line MG63/ADM. We further focused on the mechanism of action of sirolimus and directed our study toward microRNAs (miRNAs), which act as gene regulators and control complex regulatory networks by targeting mRNAs through cleavage or translational repression. We utilized high-throughput miRNA expression analysis to identify miRNAs associated with the action of sirolimus in OS cells. After exposure to sirolimus, miR-34b was significantly up-regulated. MiR-34, a tumor suppressor miRNA family, is considered to be a critical mediator of p53 function ${ }^{[12-14]}$. Several studies have demonstrated its role in resistance or sensitization to anticancer drugg ${ }^{[15,16]}$. We found two new targets of sirolimus, p21-activated protein kinase 1 (PAK1) and the $A B C B 1 / M D R 1$ genes. The current work also evaluated the relationship between miR-34b levels and the prognosis of OS. We found that drug resistance is closely related to miR-34b expression.

\section{Materials and methods}

\section{Cell culture and cell proliferation assay}

MG63/ADM and HEK 293T cells were purchased from the Chinese Academy of Sciences Cell Bank and were seeded into 96- or 6-well plates in Dulbecco's modified Eagle's medium (DMEM, Gibco, Grand Island, NY, USA) supplemented with $10 \%$ fetal bovine serum (Invitrogen, Carlsbad, CA, USA) at $37^{\circ} \mathrm{C}$ in a $5 \% \mathrm{CO}_{2}$ incubator and routinely passaged at 2- to 3 -day intervals. Doxorubicin (ADM), gemcitabine (GEM) and methotrexate (MTX) were purchased from Hengrui Medicine Co (Lianyungang, China). Sirolimus was purchased from Sigma-Aldrich (St Louis, MO, USA) and was dissolved in dimethyl sulfoxide (DMSO) at $10 \mathrm{mmol} / \mathrm{L}$ and stored frozen in aliquots. We detected the combined effect of these chemodrugs with sirolimus in MG63/ADM. For the target validation test, as soon as the HEK 293 T cells were $80 \%$ confluent, they were starved in DMEM with 1\% FBS for $24 \mathrm{~h}$ and then transfected with miR-34b-MIMIC (oligonucleotide of miR-34b) and NC (negative control miRNA) in serum-free DMEM for $6 \mathrm{~h}$. For the rescue test, miR-34b-AMO (an antagomir of miR34b) and NC were treated as described above in MG63/ADM. All nucleic acid fragments were purchased from GenePharma Biotech Company, Shanghai, China. After transfection, the MG63/ADM cells were incubated in medium containing 10\% FBS for $12 \mathrm{~h}$ and then treated with $10 \mathrm{nmol} / \mathrm{L}$ sirolimus for $24 \mathrm{~h}$. Cell proliferation assays were performed with a Cell Counting Kit-8 (CCK-8, Dojindo Molecular Technologies,
Dojindo, Japan) according to the manufacturer's instructions. The absorbance was read at $450 \mathrm{~nm}$ in a microplate reader 630 model (Bio-Rad, Hercules, CA, USA).

\section{Cell cycle analysis}

The MG63/ADM cells were treated with sirolimus and with or without miR-34b-AMO in as described above. Then, the cells were harvested by trypsinization and collected by centrifugation at $1500 \mathrm{r} / \mathrm{min}$ for $5 \mathrm{~min}$ at room temperature, after washing with phosphate-buffered saline (PBS). After being fixed in $5 \mathrm{~mL}$ of $70 \%$ ethanol at $-20^{\circ} \mathrm{C}$ overnight, the cells were washed once with PBS/1\% BSA again and then incubated at room temperature with $1 \mathrm{~mL} \mathrm{PBS/1 \%} \mathrm{BSA} \mathrm{containing} 30$ $\mu \mathrm{g} / \mathrm{mL}$ propidium iodide and $0.25 \mathrm{mg} / \mathrm{mL}$ RNase A for 30 min. The percentages of cells in different phases of the cell cycle were determined for DNA content by flow cytometry using a ModFIT system (Becton Dickinson, San Jose, CA, USA).

\section{Evaluation of cellular apoptosis}

For early and late apoptosis, the cells were treated as in the cell cycle analysis and investigated by FCM using an Annexin V-FITC Apoptosis Detection Kit (BioVision, Palo Alto, CA, USA). According to the manufacturer's protocol, Annexin V-FITC and PI ( $5 \mu \mathrm{L}$ of each) were added to $100 \mu \mathrm{L}$ of the cell suspension $\left(10^{5}\right.$ cells $\left./ \mathrm{mL}\right)$ in binding buffer. The FCM data were analyzed using the QuantiCALC system by flow cytometry (Becton Dickinson, San Jose, CA, USA).

\section{Detection of the differentially expressed miRNAs by miRNA microarray}

After treatment with or without sirolimus at $23.97 \mathrm{nmol} / \mathrm{L}$ (the $\mathrm{IC}_{50}$ that we calculated) for $24 \mathrm{~h}$, the MG63/ADM cells were harvested and subsequently analyzed using a miRNA microarray (Kangchen Bio-tech Company, Shanghai, China). Total RNA was extracted using TRIzol reagent (Invitrogen, USA). The purity and concentration of 30 to $50 \mathrm{mg}$ of the total RNA were determined with an ND-1000 spectrophotometer (NanoDrop Technologies Inc, Wilmington, DE, USA) and RNA was labeled with a miRCURY ${ }^{\mathrm{TM}}$ Hy3TM/Hy5TM Power Labeling Kit (Exiqon) and hybridized to a miRCURY ${ }^{\mathrm{TM}}$ LNA Array (Exiqon, v11.0). The results were scanned with an Axon GenePix 4000B microarray scanner and analyzed with GenePix Pro v6.0. We analyzed the miRNAs precise location in the human genome using a BLAST search (www.ncbi.nlm. nih.gov).

\section{Targeted in vitro luciferase reporter assay}

The psi-Check2 plasmid (Promega, Madison, WI, USA) was used for the expression of the miR-34b targets PAK1 3'-UTR and ABCB1 3'-UTR. The wild type (WT) Check2-PAK1 and Check2-ABCB1 constructs contained the miR-34b response element of the PAK1 and ABCB1 3'-UTR. For the mutant (MUT) Check2-PAK1 or Check2-ABCB1 constructs, we replaced two nucleotides within the seed sequences. The sequences used to create the Check2-ABCB1 constructs were as follows: for- 
ward 5'-AACTCGAGCTGCTACCTCCGCCACTT-3' (XhoI

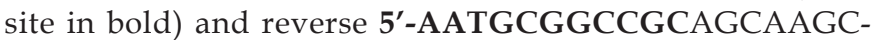
GAACAGAACAGG-3' (NotI site in bold); PAK1 forward 5'-AACTCGAGGTGTTGAGAAGTCGGGGAAAA-3' (XhoI

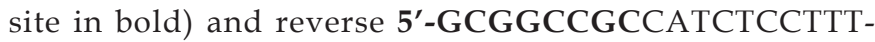
TATTGACAGAAATAGA-3' (NotI site in bold). The psiCheck2 empty vector was used as the negative control. Using the QuikChange kit (Stratagene, La Jolla, CA, USA), mutations were introduced by replacing the predicted miR-34b binding site, UGGUGCUA, with AGGAGCAA. We transfected $0.2 \mu \mathrm{g}$ of the reporter plasmid, $0.01 \mu \mathrm{g}$ of the psi-Check 2 control plas$\mathrm{mid}$, and $200 \mathrm{nmol} / \mathrm{L}$ of miR-34b-MIMIC (miR-34b precursor) and the NC miRNA (Shanghai GenePharma Company, Shanghai, China) into HEK293 T cells. Forty-eight hours later, the luciferase activity was measured using the Dual-Luciferase Reporter Assay System (Promega). Using the Renilla luciferase values as a correction factor, the ratio of Renilla/firefly values was obtained. The experiments were repeated three times with 3 parallel pores each time.

\section{qRT-PCR detection of miR-34b and its targets}

For the cultured cells, we extracted total RNA with TRIzol. For the pathologic tissue sectioning, we chose PureLink TM FFPE RNA Isolation Kit. The PCR primers were designed using Primer 3 software (http://frodo.wi.mit.edu/primer3/). Using a LightCycler480 Real-Time PCR System (Roche, Switzerland), qRT-PCR for PAK1 and ABCB1 was performed with the SYBR Green Master Mix Kit (Takara, Japan) in a reaction volume of $20 \mu \mathrm{L}$. The amplifications were conducted in triplicate for each sample. The primers were as follows: ABCB1 forward 5'-CCCATCATTGCAATAGCAGG-3' and reverse 5'-TGTTCAAACTTCTGCTCCTGA-3'; PAK1 forward 5'-AGTTTCAGAAGATGAGGATGATGA- ${ }^{\prime}$ ' and reverse $5^{\prime}$ AATCACAGACCGTGTGTATACAG- $3^{\prime}$. The results were normalized to GAPDH, which was detected using the following primers: forward 5'-GAAGGTGAAGGTCGGAGT-3' and reverse 5'-GAAGATGGTGATGGGATTTC-3'. The primer for miR-34b and its control U6 were purchased from the Jima Company. The relative quantification was calculated using the $2^{-\Delta \Delta C_{1}}$ method.

\section{Western blot analysis}

For the Western blot analysis, protein was extracted from the MG63/ADM cells using RIPA buffer in the presence of proteinase inhibitor cocktail (Shanghai Shenergy Biocolor BioScience \& Technology Company, Shanghai, China). Equal amounts of protein from of all of the groups were determined using the BCA protein assay kit (Bios, Beijing, China). Aliquots $(25 \mathrm{mg})$ were separated by $10 \%$ SDS-PAGE. Then, the proteins in the gels were transferred to nitrocellulose membranes. The membranes were probed with primary antibodies for ABCB1 (mouse monoclonal; Santa Cruz, Dallas, TX, USA) and PAK1 (goat polyclonal; Santa Cruz, Dallas, TX, USA) at room temperature for $2 \mathrm{~h}$. GAPDH (1:5000) was used as a loading control. After being washed extensively with 0.1\% Tween 20 in PBS, the membranes were incubated with the appropriate horseradish peroxide-conjugated secondary antibody at a 1:1000 dilution for $1 \mathrm{~h}$ at room temperature. The specific bands were visualized by enhanced chemiluminescence reagents. The protein bands were quantified using ImageJ software.

\section{In situ hybridization (ISH) and immunohistochemistry (IH) analysis}

According to our in vitro results, we further analyzed miR$34 \mathrm{~b}$ expression in the OS clinical samples. In this retrospective analysis approved by the Research Ethics Committee of Shanghai 6th People's Hospital, all of the specimens were derived from our sample database and follow-up visits from 2002. All of the specimens were anonymous according to the ethical and legal standards. The 40 cases of OS were divided into the following 2 groups: a group of subjects with a good response and a survival of more than 5 years $(n=17)$ and a group of subjects with a poor response, who died within three years of acquiring primary or secondary chemo-resistance. The primary chemo-resistance was associated with neo-adjuvant chemotherapy failure $(n=5)$, resulting in tumor shrinkage that was less than $25 \%$ and a tumor cell necrotic rate that was less than $10 \%$ for surgical specimens. The second chemoresistance occurred after failure of one prior chemotherapy regimen comprising ADM, DDP, MEX and IFO $(n=18)$.

For ISH, the OS tissues were fixed in $4 \%$ paraformaldehyde, dehydrated in a graded series of ethanol baths and embedded in paraffin. Then, the tissue slides were deparaffinized and digested with proteinase $\mathrm{K}$ for $30 \mathrm{~min}$. Subsequently, the slides were prehybridized in a solution at $57^{\circ} \mathrm{C}$ for $2 \mathrm{~h}$. The tissues were hybridized overnight in the presence of 10 ng of $3^{\prime}-5^{\prime}$-biotin-labeled miR-34b (Exiqon) probes at $58^{\circ} \mathrm{C}$. The slides were washed twice thoroughly, and an immunological reaction was carried out using the rabbit antibody against digoxigenin and alkaline phosphatase according to the manufacturer's recommendations. Each side was assigned a score, which ranged from $0-4$, where 0 was negative $(0 \%), 1$ was weak $(1 \%-25 \%), 2$ was medium $(26 \%-50 \%), 3$ was strong $(51 \%-75 \%)$, and 4 was strongly positive $(76 \%-100 \%)$.

To further verify the miR-34b regulation of its targets PAK1 and $A B C B 1$, we detected their protein level by $\mathrm{IH}$. IH staining was performed using the streptavidin-peroxidase (SP) method (SP kit; ZSGB-Bio, Beijing, China) according to the manufacturer's instructions. The slides were deparaffinized with xylene (BaiYi Co Ltd, Jining, China) twice for $30 \mathrm{~min}$ each, dehydrated three times in a gradient series of ethanol $(100 \%$, $95 \%, 90 \%, 80 \%$ and $70 \%$ ) and rinsed with phosphate-buffered saline (PBS). Following $15 \mathrm{~min}$ of treatment with $3 \% \mathrm{H}_{2} \mathrm{O}_{2}$, the slides were blocked using normal goat serum (Jackson ImmunoResearch, West Grove, PA, USA) for $20 \mathrm{~min}$. The slides were incubated overnight at $4{ }^{\circ} \mathrm{C}$ with primary antibodies specific to: ABCB1 (1:50 mouse monoclonal; Santa Cruz, USA) and PAK1 (1:50 goat polyclonal; Santa Cruz, USA 1). The slides were subsequently washed three times with PBS for $15 \mathrm{~min}$. The slides were treated with the SP reagent for $20 \mathrm{~min}$ and then incubated with a secondary antibody for $90 \mathrm{~min}$ at $37^{\circ} \mathrm{C}$, 
using an SP mouse and goat horseradish peroxidase kit (Cat No CW0120; CWBiotech Co Ltd, Wuhan, China) according to the manufacturer's instructions. Subsequently, the slides were washed twice with PBS for 15 min per wash and visualized using 3,3'-diaminobenzidine for $5 \mathrm{~min}$ and then counterstained with hematoxylin (Solarbio Science \& Technology Co, Ltd, Beijing, China). The slides were mounted and dried. The images were captured using an Olympus microscope (C-7070; Olympus Corporation, Tokyo, Japan). The score was calculated similarly to the ISH.

\section{Statistical analysis}

The data are expressed as the mean \pm SEM of at least three independent experiments. One-way ANOVA were used to test the differences between the means. All P-values are two-sided, and a value of 0.05 was considered to be statistically significant. All statistical calculations were performed using SPSS software (version 18.0, SPSS, USA).

\section{Results}

Sirolimus induced apoptosis, cell cycle arrest and chemosensitization in OS cells

mTOR is an established therapeutic target, and mTOR inhibitors appear to be reasonably well tolerated. The sirolimus concentration range used in this experiment was 1-100 $\mathrm{nmol} / \mathrm{L}$ for $24 \mathrm{~h}, 48 \mathrm{~h}$ and $72 \mathrm{~h}$. We calculated the $\mathrm{IC}_{50}$ each time and the weighted average of the 3 results, to calculate the final $\mathrm{IC}_{50}$ of sirolimus for MG63/ADM at $23.97 \mathrm{nmol} / \mathrm{L}$ (Figure 1A). After treatment with different concentrations of sirolimus for $24 \mathrm{~h}$, we found that it markedly induced apoptosis of the MG63/ADM cells in a dose-dependent manner (Figure 1B). Compared with the untreated control cells, sirolimus treated cells showed an increased number of cells in $G_{1}$ phase (Figure 1C). Intensive high-dose chemotherapy is a frequently used as treatment modality for OS. However, the intolerable side effects suggest that there is a significant clinical need to improve the efficacy of chemotherapy. Furthermore, at the cellular level, we used sirolimus $10 \mathrm{nmol} / \mathrm{L}$ to sensitize the MG63/ADM cells to multiple chemotherapeutic agents. As shown in Figure 1D, sirolimus significantly sensitized the cells to chemotherapy by decreasing the $\mathrm{IC}_{50}$ values of $\mathrm{ADM}, \mathrm{GEM}$ and MTX in the MG63/ADM cells from 25.48 to $4.93 \mu \mathrm{mol} / \mathrm{L}$, from 621.41 to $73.92 \mu \mathrm{mol} / \mathrm{L}$, and from 21.72 to $6.77 \mu \mathrm{mol} / \mathrm{L}$ in $24 \mathrm{~h}$.

\section{Sirolimus up-regulated miR-34b expression}

To explore the potential mechanisms of sirolimus-induced apoptosis in OS cells, we identified the miRNAs modulated by sirolimus in the MG63/ADM cells for $24 \mathrm{~h}$ using a miRNA microarray (Figure 2A). MiR-34b increased by a factor of 7.5, which represented one of the most significant and stably upregulated miRNAs as determined by qRT-PCR (Figure 2B) and it attracted our attention due to its tumor suppressor role in other cancer cells ${ }^{[17-19]}$.

\section{Effect of miR-34b on MG63/ADM}

Numerous studies have reported that miR-34 family members are tumor suppressors. We transfected MG63/ADM cells with miR-34b. Our results were consistent with those of previous studies showing that an up-regulation of miR-34b induced the chemo-sensitivity (Figure 3A) and apoptosis of the MG63/ ADM cells (Figure 3B).

\section{$A B C B 1$ and PAK1 are direct targets of miR-34b}

The miR-34s directly regulated key apoptosis and proliferation factors, such as $\mathrm{MET}^{[20]}$, $\mathrm{c}-\mathrm{Myc}^{[21]}$, and Bcl-2 ${ }^{[22]}$. However, most of these factors are regulated by miR-34a and miR-34c. In this study, miR-34b instead of miR-34a/c played a role in the effect of sirolimus on MG63/ADM. Using a TargetScan analysis, we predicted that $\mathrm{ABCB} 1$ and PAK1 contained potential miR-34b target sites in their $3^{\prime}$-UTRs. The seed sequences for miR-34b in the $3^{\prime}$-UTRs of ABCB1 and PAK1 are shown in Figure 4A. To determine the direct interaction of miR-34b with the 3'UTRs of ABCB1 and PAK1, we cloned the WT or MUT miR-34bABCB1 and PAK1 response elements into the psiR-CHECK2 plasmid downstream of the luciferase reporter and transfected HEK293T cells with a miR-34b-MIMIC and the ABCB1 and PAK1 3'UTR vectors. As shown in Figure 4B, the luciferase reporter activity of the $\mathrm{WT}$, but not the MUT Check2-ABCB1 and PAK1, was inversely correlated with the miR-34b expression level. For the WT group, luciferase reporter activity was approximately $28 \%$ to $40 \%$ of the control group $(P<0.001)$. To determine the role of ABCB1 and PAK1 downstream of miR$34 \mathrm{~b}$, we analyzed their levels by qRT-PCR (Figure 4C) and Western blotting (Figure 4D). As shown in Figure 4C and 4D, after treatment with the miR-34b-MIMIC and its AMO for $24 \mathrm{~h}$, the levels of ABCB1 and PAK1 were directly related to those of miR-34b.

\section{Inhibition of miR-34b in MG63/ADM cells reversed the effect of} sirolimus

We treated the OS cells with $10 \mathrm{nmol} / \mathrm{L}$ sirolimus with or without miR-34b-AMO transfection for $24 \mathrm{~h}$. As shown in Figure $5 \mathrm{~A}$, the down-regulation of miR-34b significantly decreased the ratio of sirolimus inhibition on MG63/ADM. Compared with the control, sirolimus reduced the expression of PAK1 and ABCB1 mRNA and protein, which was attenuated by miR-34b inhibition (Figure 5B and 5C). We then detected the apoptosis ratio in the MG63/ ADM cells. In the miR-34-AMO group, cellular apoptosis was dramatically lower than in the sirolimus group (Figure 5D). Similar trends were observed in the cell cycle analyses (Figure 5E). These results indicated that miR-34b expression plays an important role in the effects of sirolimus treatment of OS cells.

\section{Elevated miR-34b, PAK1 and $A B C B 1$ expression in OS tissue}

To elucidate the functions of miR-34b, we utilized both qRTPCR and ISH to study the expression patterns of miR-34b in OS tissues. Table 1 lists the characteristics of the 40 OS patients and their relationship with miR-34b score by the ISH method. A significantly higher miR-34b ISH score and lower $P A K 5$ and $A B C B 1$ scores were detected in the chemo-sensitive group which had a survival period lasting more than 5 years. 

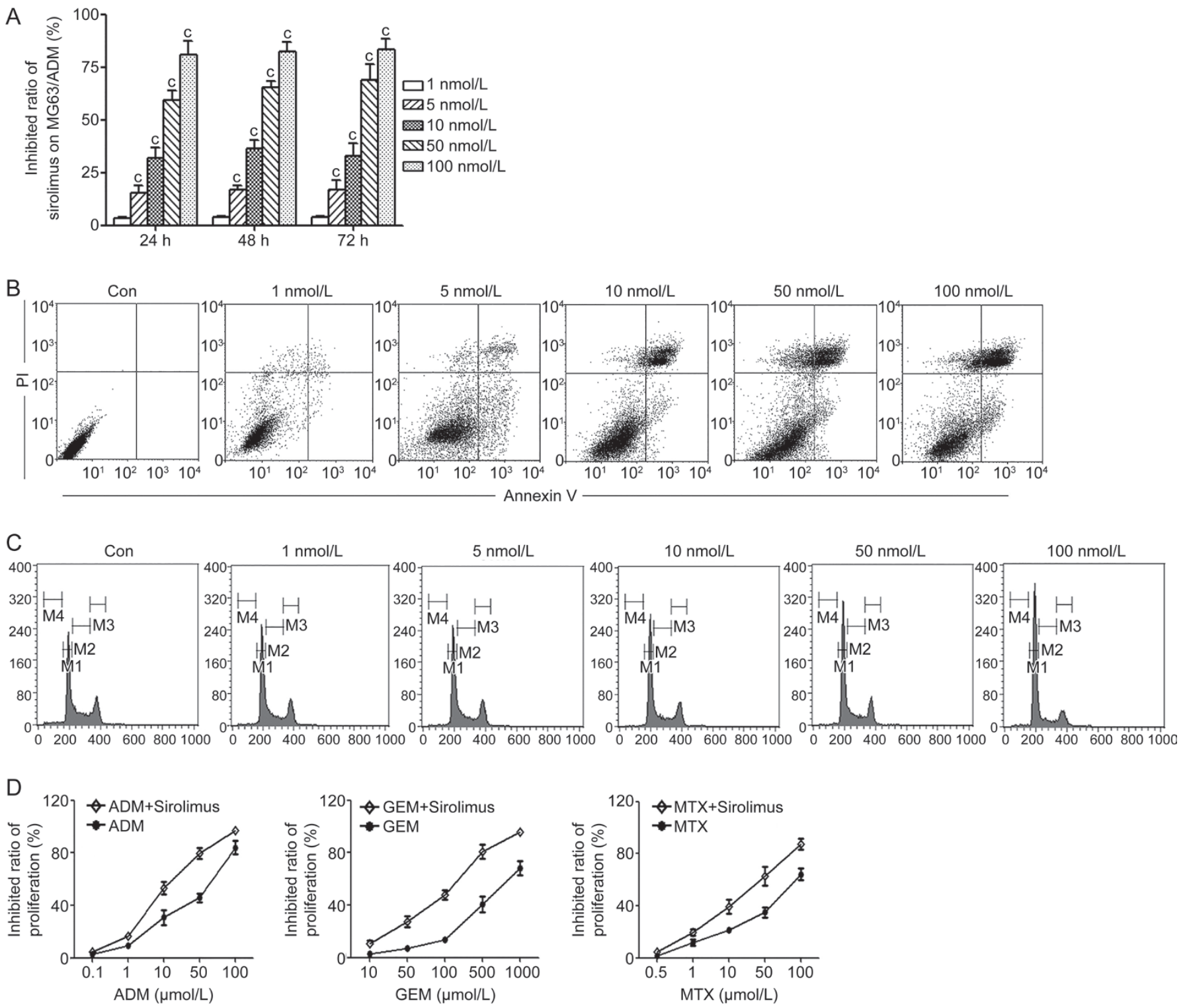

Figure 1. Sirolimus regulates the cell cycle, apoptosis and chemosensitivity in OS cells. (A) After exposure to various concentrations (from 1 to $100 \mathrm{nmol} / \mathrm{L}$ ) of sirolimus for 24,48 and $72 \mathrm{~h}$, the cell viability of MG63/ADM was determined using the CCK-8 assay. The untreated group served as a control. The $\mathrm{Y}$-axis, the inhibited ratio, was calculated according to the formula 1-(OD of Experimental/OD of Control) $\left({ }^{\circ} P<0.01\right.$ compared with the untreated control group). (B) The MG63/ADM cells were treated with 1-100 nmol/L sirolimus for $24 \mathrm{~h}$. The apoptotic cells were stained with annexin V/PI and analyzed using FACS. (C) The MG63/ADM cells were treated as in (B) but were stained only with PI, and the cell cycle distribution was determined using FACS; the number of cells in the $G_{1}$ phase increased in a dose-dependent manner. (D) Exposure to $10 \mathrm{nmol} / \mathrm{L}$ sirolimus elevated the sensitivity to ADM, GEM and MTX in the MG63/ADM cells.

Compared with the two groups, a low expression of miR-34b was significantly associated with the tumor stage and necrosis but not with other clinical parameters. The qRT-PCR results were similar to the ISH and IH results (Figure 6A). We examined the correlation between miR-34b and its targets. MiR-34b was significantly negatively correlated with PAK1 and ABCB1. The correlation coefficients were -0.710 and -0.764 , and all the $P<0.001$.

\section{Discussion}

As the most frequent malignant primary bone tumor, osteo- sarcoma is highly aggressive and metastatic at a very early stage. Prior to adjuvant and neoadjuvant chemotherapy, the long-term survival rate subsequent to surgical resection alone is $<20 \%$. Multi-drug chemotherapy regimens have dramatically improved the survival rate, to up to $50 \%$ to $70 \%{ }^{[23]}$. However, multiple drug resistance is an undesirable outcome. Cell signaling induced by cytokines/growth factors occurs via the $\mathrm{PI} 3 \mathrm{~K} / \mathrm{mTOR}$ pathway, including multiple drug resistance ${ }^{[24]}$. Sirolimus, the first mTOR inhibitor, combined with various targeted biologic agents or cytotoxic chemotherapies is under investigation in more than 40 clinical trials and has shown 
A

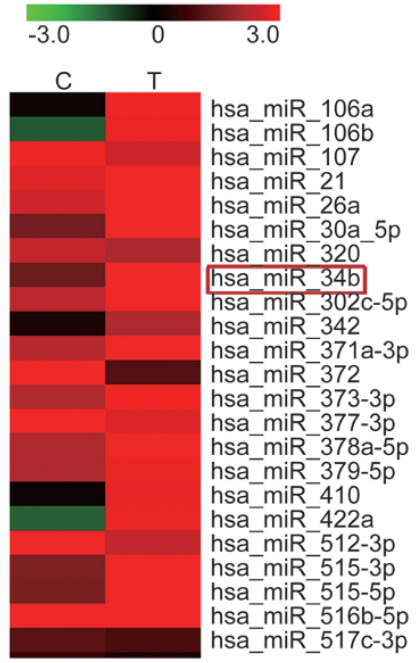

$\mathrm{B}$

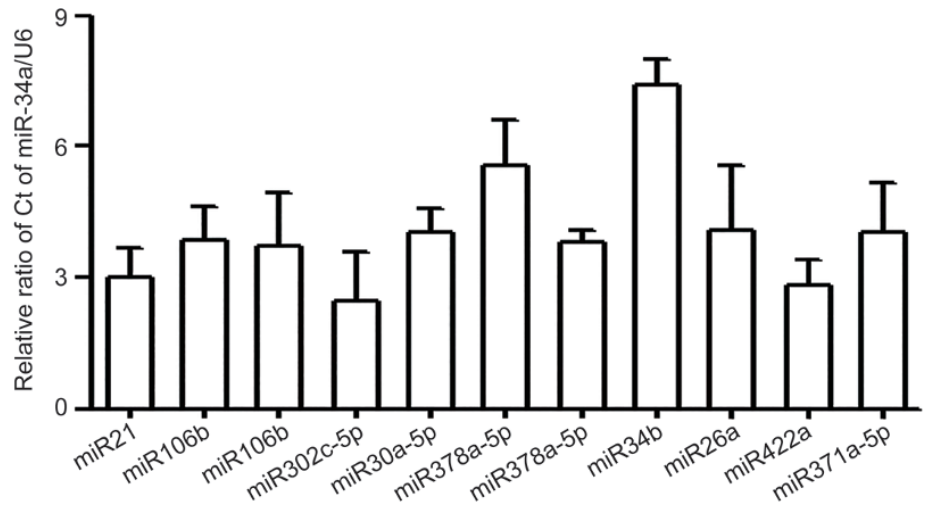

Figure 2. Sirolimus up-regulates miR-34b. (A) Microarray analysis was used to compare the expression profiles of 703 miRNAs in the MG63/ADM cells that were untreated (labeled as C) or treated (labled as T) with $23.97 \mathrm{nmol} / \mathrm{L}$ sirolimus. MiR-34b, one of the most markedly up-regulated miRNAs, is labeled with a red box. (B) qRT-PCR detection of 11 miRNAs in the MG63/ADM cells treated with sirolimus showed dramatically increased miR-34b levels (7.5).

A

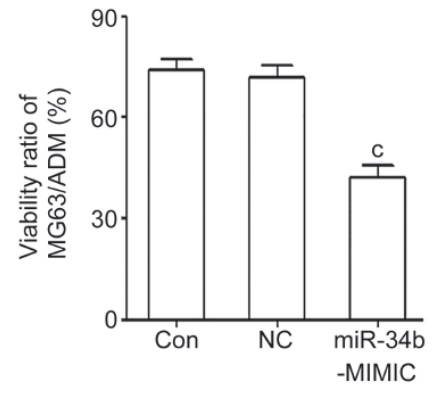

ADM

B

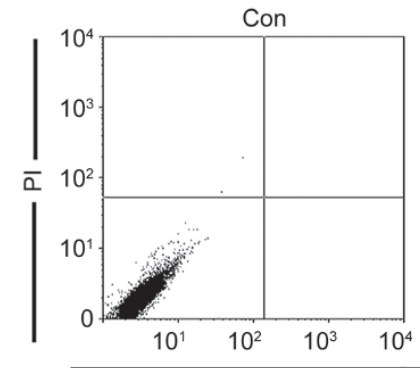

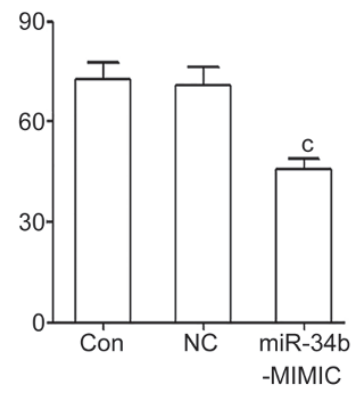

GEM

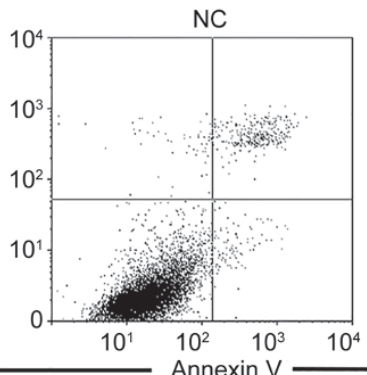

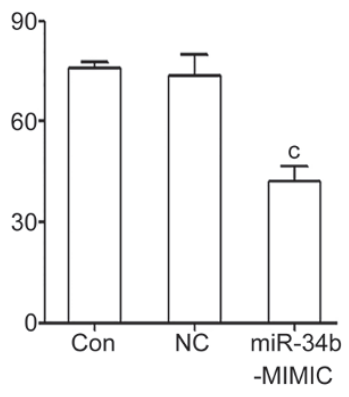

MTX

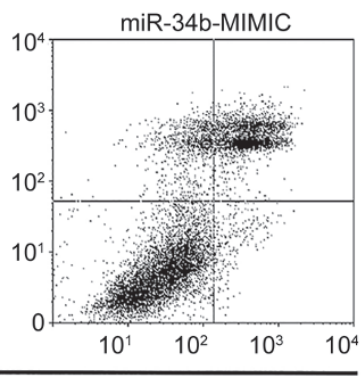

Figure 3. Effect of miR-34b. (A) After transfection with the miR-34b-MIMIC for $24 \mathrm{~h}$, we treated the MG63/ADM cells with a $1 / 2 \mathrm{IC}_{50}$ concentration of ADM, GEM and MTX, and the cell viability of MG63/ADM was determined using the CCK-8 assay. MiR-34b also elevated the sensitivity to chemotherapy. The Y-axis, the viability ratio, was calculated according to the formula 1-(OD of Experimental $/ O D$ of Control) ( ${ }^{\circ} P<0.01$ compared with the untreated control group). (B) The MG63/ADM cells were treated with the miR-34b-MIMIC or NC for $24 \mathrm{~h}$. The apoptotic cells were stained with annexin V/PI and analyzed using FACS.

early promising results ${ }^{[25,26]}$. Sirolimus inhibits the growth of the MDR leukemic cell lines in vitro by modifying the expression of mTOR. It effectively suppresses the phosphorylation of two downstream effector molecules in the mTOR pathway, S6K1 and 4E-BP1. Sirolimus has been shown to modulate the expression and function of P-gp and to chemosensitize cell lines as effectively as verapamil ${ }^{[27]}$. However, the efficacy of mTOR inhibitors alone in cancer treatment is only modest. These inhibitors may not completely inhibit mTOR function because they interfere with only the function of the mTOR/raptor (regulatory-associated protein of mTOR) complex and mTOR kinase inhibitors ${ }^{[28]}$. Therefore, we studied sirolimus treat- 


\section{A}

Position 276-301 of ABCB1 3'-UTR 5 ...AUUUGGACUGUAACUGACUGCCUU.. (Conserved) hsa-miR-34b 3 ....GUUAGUCGAU-UACUGUGACGGAU...

Position 849-875 of PAK1 3'-UTR 5 ...AACUCAGCCCCUUUUUCUGCCUAG... (Nonconserved) hsa-miR-34b 3 ...GUUAGUC-GAU-UACUGUGACGGAU...

Position 1185-1209 of PAK1 3'-UTR 5 ...AUGCUCUGAGGUACUACUGCCUC... (Conserved) hsa-miR-34b 3 ...GUUAGUCGAUUACUGUGACGGAU...

B

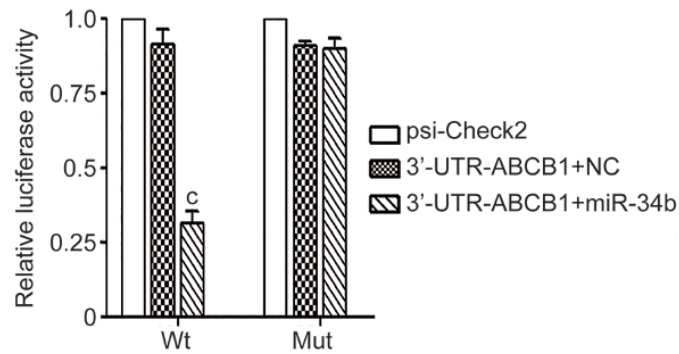

C

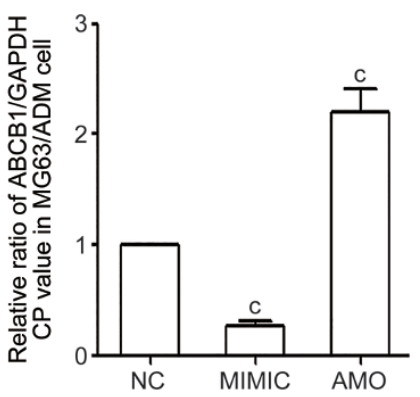

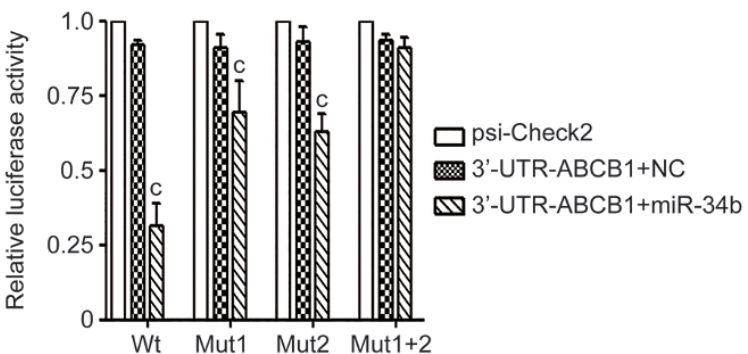

D
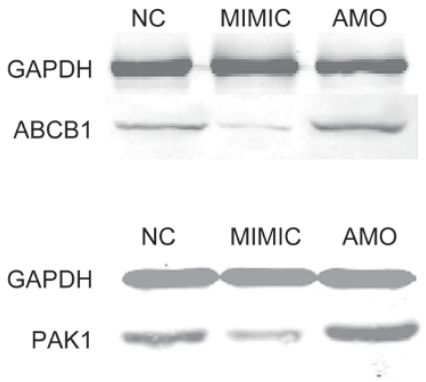

Figure 4. Analysis of miR-34b target sites. (A) The selection criteria of the miRNA targets were based on their common detection in the target prediction online databases (TargetScan5.1 http://www.targetscan.org) as well as the full complementarity between the seed region of miR-34b and the 3'UTRs of PAK1 and ABCB1. (B) HEK 293T cells were co-transfected with the miR-34b-MIMIC, psi-Check2, WT-psi-Check2 or MUT-psi-Check2 of PAK1 and ABCB1 genes. The luciferase activity levels were measured $48 \mathrm{~h}$ after transfection. The results from at least three independent experiments are presented as the mean \pm SEM. In this panel, the luciferase assay results show the regulation of PAK1 and ABCB1 by miR-34b ( ${ }^{\circ} P<0.01$ compared with the control). (C) The PAK1 and ABCB1 mRNA expression levels in the MG63/ADM cells transfected with the miR-34-MIMIC or miR-34b-AMO were determined by qRTPCR ( ${ }^{\circ} P<0.01$ compared with the control). (D) The PAK1 and ABCB1 protein expression levels in the MG63/ADM cells transfected with the miR-34bMIMIC or miR-34b-AMO were examined by a Western blot analysis.

Table 1. Analyisis of miR-34b expression and the clinical character of OS sample.

\begin{tabular}{|c|c|c|c|c|}
\hline & & Chemo-sensitivity & Chemo-resistance & $P$ \\
\hline \multirow[t]{2}{*}{ Age } & $<15$ & 10 & 18 & 0.194 \\
\hline & $\geq 15$ & 7 & 5 & \\
\hline \multirow[t]{2}{*}{ Gender } & Male & 9 & 13 & 0.827 \\
\hline & Female & 8 & 10 & \\
\hline \multirow[t]{2}{*}{ Anatomic location } & Tibia/femur & 10 & 11 & 0.504 \\
\hline & Elsewhere & 7 & 12 & \\
\hline \multirow[t]{2}{*}{ Tumor size $(\mathrm{cm})$} & $\leq 8$ & 11 & 12 & 0.093 \\
\hline & $>8$ & 6 & 11 & \\
\hline \multirow[t]{2}{*}{ Clinical stage } & IIA & 12 & 8 & 0.025 \\
\hline & IIB/III & 5 & 15 & \\
\hline \multirow[t]{2}{*}{ Necrosis rate } & $\leq 25 \%$ & 3 & 11 & 0.049 \\
\hline & $>25 \%$ & 14 & 12 & \\
\hline \multirow[t]{2}{*}{ Pathological type } & Conventional & 11 & 15 & 0.974 \\
\hline & Non-convention & 6 & 8 & \\
\hline miR-34b & Score & 29 & 5 & $<0.001$ \\
\hline PAK1 & Score & 26 & 48 & $<0.001$ \\
\hline ABCB1 & Score & 20 & 63 & $<0.001$ \\
\hline
\end{tabular}




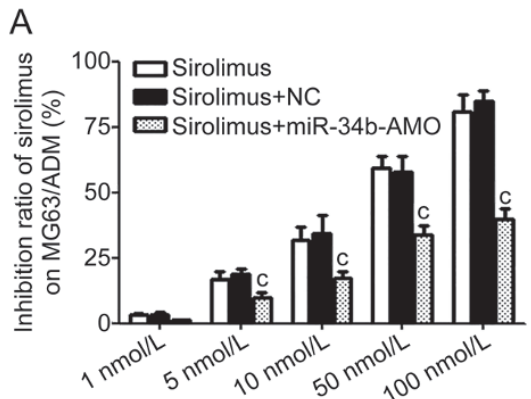

B

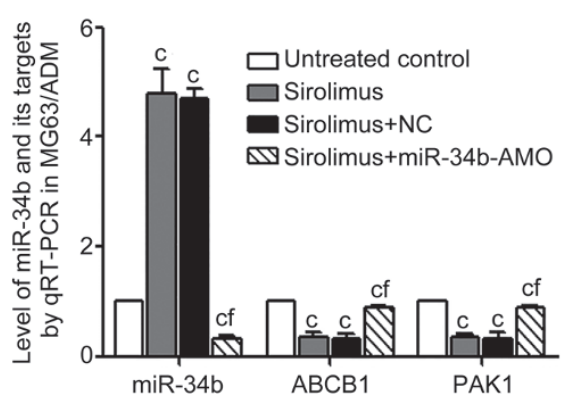

C

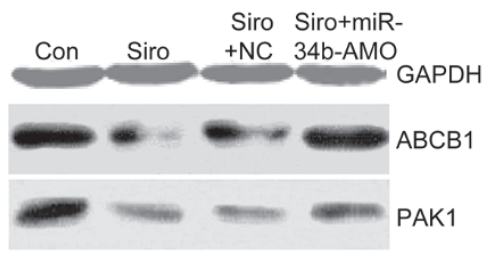

D

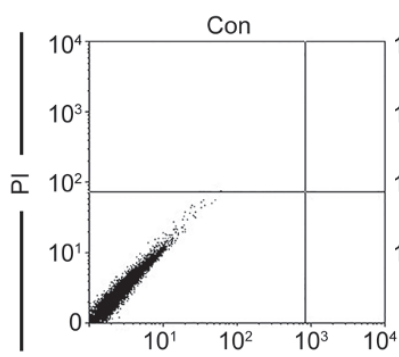

$10 \mathrm{nmol} / \mathrm{L}$ Sirolimus
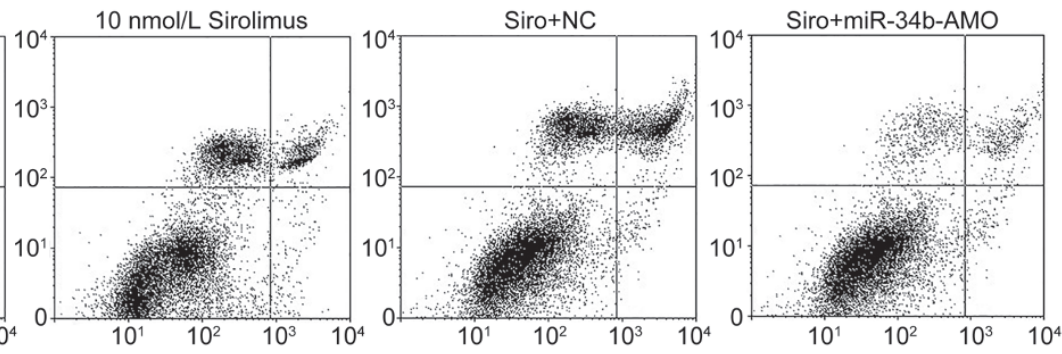

-Annexin V

E
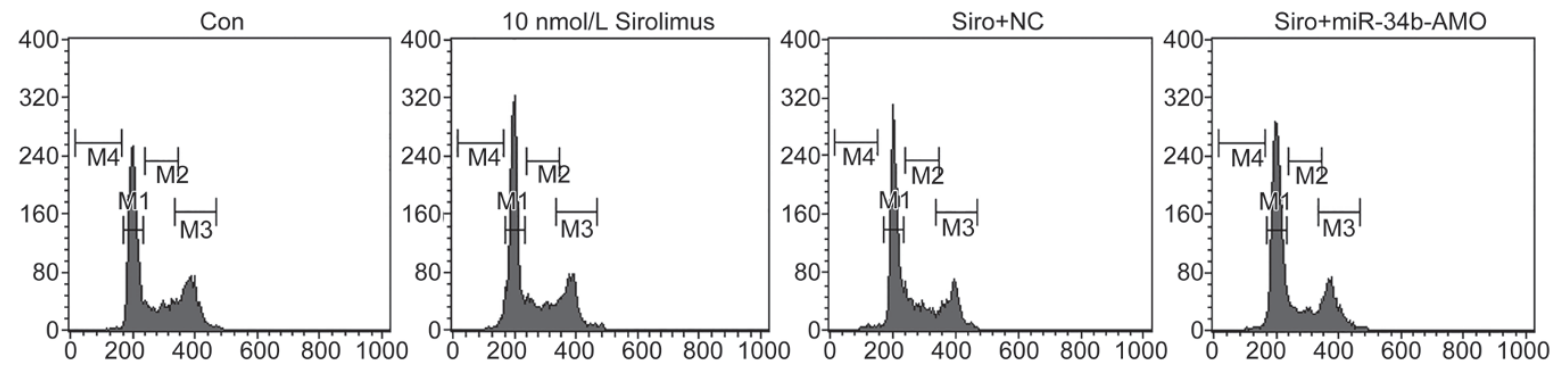

Figure 5. miR-34-AMO reversed the effect of sirolimus. miR-34-AMO reversed the effects of sirolimus (10 nmol/L) on the MG63/ADM cells. In this figure, all of the cells were treated under similar conditions. After transfer of miR-34-AMO or NC for $12 \mathrm{~h}$, the cells were exposed to $10 \mathrm{nmol} / \mathrm{L}$ sirolimus for $24 \mathrm{~h}$. (A) The cell viability of MG63/ADM was determined using the CCK-8 assay. The Y-axis, the inhibited ratio, was calculated according to the formula 1-(OD of Experimental/OD of Control) $\left({ }^{\circ} P<0.01\right.$ compared with the control group). (B) The mRNA levels of miR-34b, $P A K 1$ and $A B C B 1$ in the MG63/ADM cells were detected by qRT-PCR. The Y-axis is the relative level of miR-34b and its targets PAK1 and ABCB1, and the ratio was calculated according to the $2^{-\Delta \Delta C t}$ method. $U 6$ is the internal control of miR-34b, and $\beta$-actin is the internal control of PAK1 and ABCB1 ( ${ }^{\mathrm{C}} P<0.01$ compared with the control group; ${ }^{f} P<0.01$ compared with the sirolimus+NC group). (C) The protein levels of PAK1 and ABCB1 in MG63/ADM were detected by Western blotting. (D) After regulating miR-34b, changes in the apoptotic ratio of MG63/ADM were detected by FCM. (E) Cell cycle alterations in the MG63/ADM cells were detected.

ment in combination with other anticancer agents to achieve better therapeutic outcomes in OS patients. The present study evaluated the in vitro cytotoxic activity of $10 \mathrm{nmol} / \mathrm{L}$ sirolimus in combination with doxorubicin, gemcitabine and methotrexate. We clearly showed the concentration-dependent effects of sirolimus on growth inhibition and apoptosis in MG63/ADM. We found that sirolimus significantly sensitized cells to chemotherapy by decreasing the $\mathrm{IC}_{50}$ values of $\mathrm{ADM}, \mathrm{GEM}$ and MTX in the MG63/ADM cells. Studies have suggested that miRNAs modulate gene expression at the posttranscriptional level. Therefore, we hypothesized that microRNAs mediate sirolimus-induced apoptosis to overcome drug resistance. Microarray platforms enable high-throughput miRNA profiling and allowed us to identify miRNAs with altered expres- sion after treatment with sirolimus. Our data provide results obtained by diverse miRNAs using microarray analysis. Concordantly with the qRT-PCR results, miR-34b increased most markedly and stably. This miRNA regulates the ABCB1 and is designated as MRP1. We hypothesized that sirolimus reverses resistance to chemotherapy. The relationship between miR34 and chemo-sensitivity has never been reported before. The role of miR-34 in OS has been reported by only a few studies. Recent studies have revealed that the miR-34 family mediates apoptosis and the cell cycle via repression of proteins involved in the regulation of these two biological processes. The miR-34 family is a direct target of the tumor suppressor gene $\mathrm{p} 53^{[29]}$. Up-regulation of miR-34 induces apoptosis, cell cycle arrest and senescence, directly targeting a considerable 
A
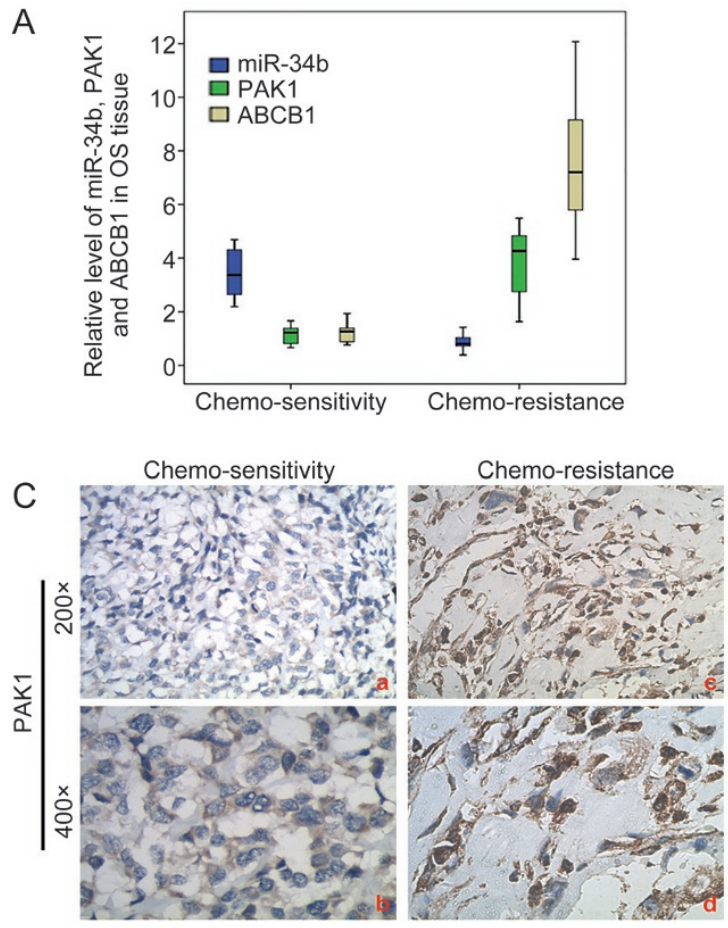

B
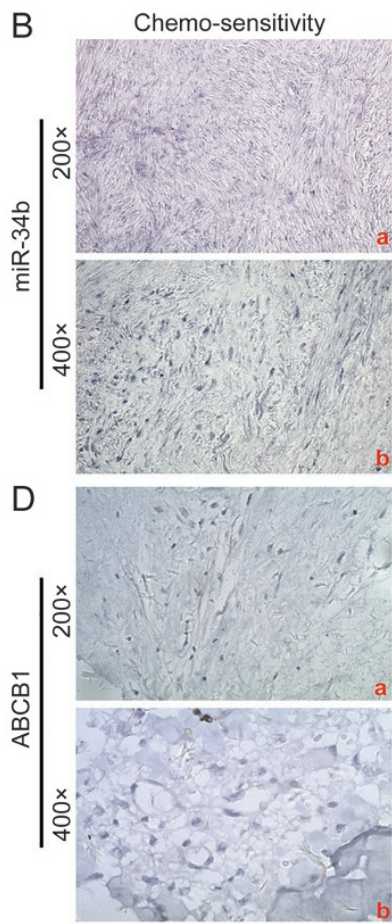

Chemo-resistance
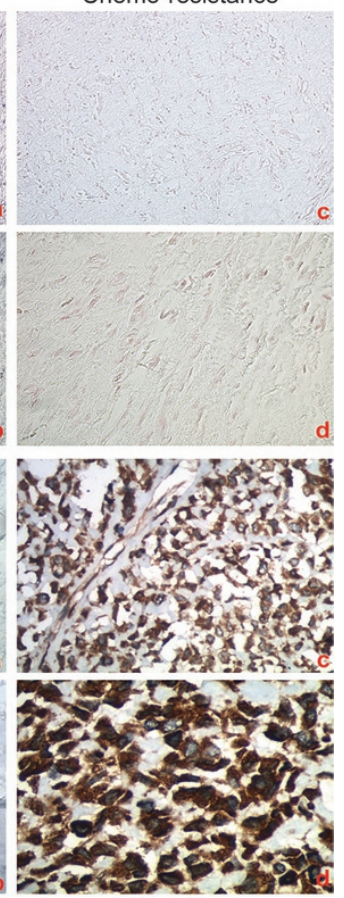

Figure 6. Detection of miR-34b expression in OS tissue. In situ hybridization analyses using 5'-biotin-labeled DNA probes complementary to miR-34b were performed. Positive ISH staining appears as blue-violet. (A) represents the expression of miR-34b, PAK1 and ABCB1 levels in OS tissue detected by qRT-PCR. (B) represents the miR-34b levels in the OS samples: $a$ ) and b) indicate that the expression of miR-34b was positive in the OS tissue with a survival exceeding 5 years, visualized under 200 and 400 vision fields, respectively; c) and d) indicate the expression of miR-34b was almost negative in the primary chemo-resistant tissue, visualized under 200 and 400 vision fields, respectively. (C) represents the expression of PAK1 in OS tissue: a) and b) are weak yellow in the cell plasma; c) and d) indicate the expression of PAK1 in the primary chemo-resistant tissue, visualized under 200 and 400 vision fields, respectively. It is strongly positive. (D) represents the ABCB1 levels in the OS tissue: $a$ ) and b) are almost negatively stained in the chemosensitive group; c) and d) show the claybank particles distributed alone in the cell membrane, visualized under 200 and 400 vision fields, respectively.

number of oncogenes such as Bcl-2, P21, CDK4, CDK6, SNAIL (epithelial mesenchymal transition) ${ }^{[30]}$ and CD44 (migration and metastasis $)^{[31]}$. We also found that after prolonged transfection of miR-34b, sirolimus increased chemo-sensitivity via unknown mechanisms. In the present study, we clarified that sirolimus induces apoptosis and reverses multidrug resistance through miR-34b by negatively regulating ABCB1 and PAK1. The p21-activated kinases (Pak) are serine/threonine protein kinases, which are oncogene activators of the small GTPases Rac1 and Cdc42, particularly in cell proliferation, apoptosis and cell cycle arrest. PAK1 is also one of the downstream proteins in the mTOR/P70S6K pathway. p70 S6 kinase activates PAK1 and contributes to phosphatidylinositol 3-kinase- and ERK-mediated regulation of HCV RNA replication, the phenomena observed were abrogated by rapamycin treatment ${ }^{[32]}$. PAK1 is associated with aggressive breast cancer ${ }^{[33]}$, colorectal cancer ${ }^{[34]}$, pancreatic cancer and other cancers. The miRNAs negatively modulate target genes by post-transcriptional regulation. A target scan analysis revealed that the $3^{\prime}$-UTRs of $A B C B 1$ and PAK1 retain binding sites for miR-34b. Of the two binding sites between PAK1 and miR-34b, only one is conserved. The luciferase assays using the ABCB1 and PAK1 3'-UTR reporter plasmids in HEK293T cells showed that luciferase activity decreased with the overexpression of miR-34b, and the deletion of the miR-34b binding site within the ABCB1 and PAK1 3'-UTRs partly abolished the decreased luciferase activity. Compared with the conserved binding site of PAK1, mutating the non-conserved site decreased the luciferase activity more slowly over a wide range due to the instability associated with a six- rather than a seven- or eight-DNA-fragment complementation. We investigated whether silencing miR$34 \mathrm{~b}$ affected the role of sirolimus. The transfection of the miR34-AMO into the MG63/ADM cells significantly reversed the effects of sirolimus on cell cycle arrest, apoptosis and chemosensitivity.

To examine the prognostic implications of miR-34b expression, we analyzed 40 cases of OS tissue samples retrospectively using the ISH method. The results revealed that in all of the 17 chemo-sensitive OS cases with more than 5 years of survival, miR-34b-positive expression was detected in the neoplastic cells with a score of 2 to 3 . However, no strongly positive case was found. The other 23 cases with primary or secondary chemo-resistance marked by death were negative or significantly weakly positive. Previous studies have shown that miR-34 family members act as tumor suppressors. MiR34 has been suggested as a new weapon against cancer ${ }^{[35]}$. MiR-34 expression is induced by severe stress, such as radiation therapy ${ }^{[36]}$ and chemotherapy. In esophageal squamous 
carcinoma, the expression level of miR-34a, induced by the DNA damage agent adriamycin (ADR), is both p53- and timedependent ${ }^{[37]}$, suggesting that miR-34b may be an oncogenic response. The up-regulation of $\mathrm{miR}-34 \mathrm{~b}$ controls cancer progression. Low miR-34 is a poor prognostic biomarker. The targets of miR-34b, including PAK1 and ABCB1, showed a contrasting trend with overexpression in chemo-resistance. MiR-34b was significantly negatively correlated with PAK1 and ABCB1. The correlation coefficients were -0.710 and -0.764 , respectively.

In conclusion, our study clearly demonstrates that sirolimus negatively regulated the expression of PAK1 and ABCB1 or $\mathrm{MRP} / \mathrm{P}-\mathrm{gp}$ by elevating miR-34b. This study shows that miR$34 \mathrm{~b}$ negatively regulates the expression and function of P-gp via repression of mRNA by directly acting on the $3^{\prime}$-UTR of ABCB1 mRNA.

\section{Acknowledgements}

This work was supported by grants from National Natural Science Foundation of China (№ 81172548 and 81372873).

\section{Author contribution}

Da-liu MIN designed research; Yan ZHOU performed research; Rui-hua ZHAO finished ISH and IH tests; Kuo-Fu TSENG contributed analytic tools; Kun-peng LI provided new reagents; Zhi-gang LU collected the cases; Yuan LIU made the pathological section; Kun HAN follwed-up the cases; Zhi-hua GAN analyzed data; Shu-chen LIN polishes the English translation; Hai-yan HU wrote the paper.

\section{References}

1 Jaffe N. Osteosarcoma: review of the past, impact on the future. The American experience. Cancer Treat Res 2009; 152: 239-62.

2 Milosevic Z, Pesic M, Stankovic T, Dinic J, Milovanovic Z, Stojsic J, et al. Targeting RAS-MAPK-ERK and PI3K-AKT-mTOR signal transduction pathways to chemosensitize anaplastic thyroid carcinoma. Transl Res 2014; 164: 411-23.

3 Zhang H, Cohen AL, Krishnakumar S, Wapnir IL, Veeriah S, Deng G, et al. Patient-derived xenografts of triple-negative breast cancer reproduce molecular features of patient tumors and respond to mTOR inhibition. Breast Cancer Res 2014; 16: R36.

4 Utomo WK, Narayanan V, Biermann K, van Eijck CH, Bruno MJ. mTOR is a promising therapeutical target in a subpopulation of pancreatic adenocarcinoma. Cancer Lett 2014; 346: 309-17.

5 Okazaki H, Matsunaga N, Fujioka T, Okazaki F, Akagawa Y, Tsurudome Y, et al. Circadian regulation of mTOR by the ubiquitin pathway in renal cell carcinoma. Cancer Res 2014; 74: 543-51.

6 Arceci RJ, Stieglitz K, Bierer BE. Immunosuppressants FK506 and rapamycin function as reversal agents of the multidrug resistance phenotype. Blood 1992; 80: 1528-36.

7 Wang Y, Hu Z, Liu Z, Chen R, Peng H, Guo J, et al. MTOR inhibition attenuates DNA damage and apoptosis through autophagy-mediated suppression of CREB1. Autophagy 2013; 9: 2069-86.

8 Zhao S, Lu N, Chai Y, Yu X. Rapamycin inhibits tumor growth of human osteosarcomas. J BUON 2015; 20: 588-94.

9 Ajabnoor GM, Crook T, Coley HM. Paclitaxel resistance is associated with switch from apoptotic to autophagic cell death in MCF-7 breast cancer cells. Cell Death Dis 2012; 3: e260.
10 McCarty MF. mTORC1 activity as a determinant of cancer risk rationalizing the cancer-preventive effects of adiponectin, metformin, rapamycin, and low-protein vegan diets. Med Hypotheses 2011; 77: 642-8.

11 Paoloni MC, Mazcko C, Fox E, Fan T, Lana S, Kisseberth W, et al. Rapamycin pharmacokinetic and pharmacodynamic relationships in osteosarcoma: a comparative oncology study in dogs. PLoS One 2010; 5: e11013.

12 Navarro F, Lieberman J. miR-34 and p53: new insights into a complex functional relationship. PLoS One 2015; 10: e0132767.

13 Li W, Han W, Ma Y, Cui L, Tian Y, Zhou Z, et al. P53-dependent miRNAs mediate nitric oxide-induced apoptosis in colonic carcinogenesis. Free Radic Biol Med 2015; 85: 105-13.

14 Rokavec M, Li H, Jiang L, Hermeking $\mathrm{H}$. The p53/miR-34 axis in development and disease. J Mol Cell Biol 2014; 6: 214-30.

15 Siemens H, Jackstadt R, Kaller M, Hermeking H. Repression of c-Kit by p53 is mediated by miR-34 and is associated with reduced chemoresistance, migration and stemness. Oncotarget 2013; 4: 1399-415.

16 Bao B, Li Y, Ahmad A, Azmi AS, Bao G, Ali S, et al. Targeting CSCrelated miRNAs for cancer therapy by natural agents. Curr Drug Targets 2012; 13: 1858-68.

17 Kim CH, Kim HK, Rettig RL, Kim J, Lee ET, Aprelikova O, et al. miRNA signature associated with outcome of gastric cancer patients following chemotherapy. BMC Med Genomics 2011; 4: 79.

18 Wang L, Yu J, Xu J, Zheng C, Li X, Du J. The analysis of microRNA-34 family expression in human cancer studies comparing cancer tissues with corresponding pericarcinous tissues. Gene 2014; 554: 1-8.

19 Zhao J, Lammers P, Torrance CJ, Bader AG. TP53-independent function of miR-34a via HDAC1 and p21 (CIP1/WAF1). Mol Ther 2013; 21: 1678-86.

20 Wang LG, Ni Y, Su BH, Mu XR, Shen HC, Du JJ. MicroRNA-34b functions as a tumor suppressor and acts as a nodal point in the feedback loop with Met. Int J Oncol 2013; 42: 957-62.

21 Cannell IG, Bushell M. Regulation of Myc by miR-34c: A mechanism to prevent genomic instability? Cell Cycle 2010; 9: 2726-30.

22 Tanaka N, Toyooka S, Soh J, Tsukuda K, Shien K, Furukawa M, et al. Downregulation of microRNA-34 induces cell proliferation and invasion of human mesothelial cells. Oncol Rep 2013; 29: 2169-74.

23 Anderson ME. Update on survival in osteosarcoma. Orthop Clin North Am 2016; 47: 283-92.

24 Martz CA, Ottina KA, Singleton KR, Jasper JS, Wardell SE, PerazaPenton A, et al. Systematic identification of signaling pathways with potential to confer anticancer drug resistance. Sci Signal 2014; 7: ra121.

25 Liesveld JL, O'Dwyer K, Walker A, Becker MW, Ifthikharuddin JJ, Mulford D, et al. A phase I study of decitabine and rapamycin in relapsed/refractory AML. Leuk Res 2013; 37: 1622-7.

26 Moroney J, Fu S, Moulder S, Falchook G, Helgason T, Levenback C, et al. Phase I study of the antiangiogenic antibody bevacizumab and the mTOR/hypoxia-inducible factor inhibitor temsirolimus combined with liposomal doxorubicin: tolerance and biological activity. Clin Cancer Res 2012; 18: 5796-805.

27 Pop IV, Pop LM, Ghetie MA, Vitetta ES. Targeting mammalian target of rapamycin to both downregulate and disable the P-glycoprotein pump in multidrug-resistant B-cell lymphoma cell lines. Leuk Lymphoma 2009; 50: 1155-62.

28 Noh WC, Mondesire WH, Peng J, Jian W, Zhang H, Dong J, et al. Determinants of rapamycin sensitivity in breast cancer cells. Clin Cancer Res 2004; 10: 1013-23.

29 Ramaiah MJ, Pushpavalli SN, Lavanya A, Bhadra K, Haritha V, Patel 
$\mathrm{N}$, et al. Novel anthranilamide-pyrazolo [1,5-a] pyrimidine conjugates modulate the expression of p53-MYCN associated micro RNAs in neuroblastoma cells and cause cell cycle arrest and apoptosis. Bioorg Med Chem Lett 2013; 23: 5699-706.

30 Brabletz T. MiR-34 and SNAIL: another double-negative feedback loop controlling cellular plasticity/EMT governed by p53. Cell Cycle 2012; 11: $215-6$.

31 Jin K, Xiang Y, Tang J, Wu G, Li J, Xiao H, et al. miR-34 is associated with poor prognosis of patients with gallbladder cancer through regulating telomere length in tumor stem cells. Tumour Biol 2014; 35: 1503-10.

32 Ishida H, Li K, Yi M, Lemon SM. p21-activated kinase 1 is activated through the mammalian target of rapamycin/p70 S6 kinase pathway and regulates the replication of hepatitis $C$ virus in human hepatoma cells. J Biol Chem 2007; 282: 11836-48.

33 Hammer A, Diakonova M. Tyrosyl phosphorylated serine-threonine kinase PAK1 is a novel regulator of prolactin-dependent breast cancer cell motility and invasion. Adv Exp Med Biol 2015; 846: 97-137.

34 Qing $H$, Gong W, Che Y, Wang X, Peng L, Liang Y, et al. PAK1dependent MAPK pathway activation is required for colorectal cancer cellproliferation. Tumour Biol 2012; 33: 985-94.

35 Maroof H, Salajegheh A, Smith RA, Lam AK. Role of microRNA-34 family in cancer with particular reference to cancer angiogenesis. Exp Mol Pathol 2014; 97: 298-304.

36 Balça-Silva J, Sousa Neves S, Gonçalves AC, Abrantes AM, CasaltaLopes J, Botelho MF, et al. Effect of miR-34b overexpression on the radiosensitivity of non-small cell lung cancer cell lines. Anticancer Res 2012; 32: 1603-9.

37 Ye Z, Fang J, Dai S, Wang Y, Fu Z, Feng W, et al. MicroRNA-34a induces a senescence-like change via the down-regulation of SIRT1 and up-regulation of p53 protein in human esophageal squamous cancer cells with a wild-type p53 gene background. Cancer Lett 2016; 370: 216-21. 